\title{
1. Einleitendes
}

Seit 70 Jahren ist die Anfrage des Josef Ben Jehuda an seinen Lehrerlaimonides, ob die Lebensdauer des Menschen fest und unabänderlich bestimmt sei, oder ob man sie durch Ergreifen von VorsichtsmaBnahmen verlängern könne, bekannt, und es ist sonderbar, daB der Originaltext der Antwort des Maimondes erst jetzt der wissenschaftlichen Welt zugänglich gemacht wird.

Im Jahre 1879 machte Moritz Steinschneider in seiner Zeitschrift «Hebräische Bibliographie» (Bd. 19, S. 131) davon Mitteilung, daB der Buch- und Handschriftenhändler Shapfra von einem gefahrvollen Aufenthalt im Jemen gegen 120 zum Teil recht wertvolle hebräische und arabische Handschriften nach Europa gebracht habe. Unter den Manuskripten, die Steinschneider vorgelegen haben und von ihm durchgesehen worden sind, befanden sich auch zwei kleine Bände, die in den Kauflisten von Shapira die Nummern 102 und 103 trugen, und die verschiedene arabische Kommentare zum Mishne Tora enthielten. Als besonders wichtig hebt Steinschneider Nr. 102 hervor, weil in ihm bei der Erklärung zu Abschnitt 10 der Hilkhót Yesódé hat-tórü ein unbekanntes Responsum des Maimonides auszugsweise mitgeteilt werde, das er seinem Schüler auf die oben angeführte Frage erteilt hätte. In Nr. 103 werde auf fol. 83b noch einmal auf diese wichtige Antwort des Maimondes hingewiesen.

Steinschneider hat sich, wie er an der angegebenen Stelle der «Hebräischen Bibliographie» berichtet, im Jahre 1879 eine Kopie von diesem Responsum des Maimondes gemacht, jedoch nur eine unzureichende Abschrift des nicht immer deutlich geschriebenen Origina1s; denn neun Jahre später hat er in Berliners «Magazin für die Wissenschaft des Judentums» (Jahrg. 15, 1888, 5.110) in einem Aufsatz über Josef ibn Aknin nur dessen kurze Anfrage ohne den Text des Responsums zum Abdruck gebracht, und auch die Anfrage in einer Form, die einige Jahre später AnlaB zur Kritik gab. Denn als David Kaufmann im Jahre 1895 in der Zeitschrift der Deutschen Morgenländischen Gesellschaft (Bd. 49, S. '73 ff.) über ein Responsum handelte, das anderthalb Jahrhunderte vor Maimondes der babylonische Gaon Hai in derselben Frage der Lebensdauer erteilt hatte, wies er auf diese kurze Mitteilung Steinschneiders hin, bemerkte 
«ohne handschriftliche Vorlage kaum völlig zu heilen» sei. Merkwürdig bleibt jedoch, daß Kaufmann sich nicht darum bemüht hat, diese handschriftliche Vorlage in die Hände zu bekommen, um den verderbten Text zu verbessern, was damals durch mündliche Nachfrage sehr leicht gewesen wäre. Auch in den folgenden Jahrzehnten hat sonderbarerweise niemand etwas unternommen, um das Original des Responsums des Maimondes zu finden und herauszugeben. Durch einen Zufall fiel mir im Jahre 1925 eine Notiz in die Hand, die ich mir als junger Student im Jahre 1902 nach einem Besuche bei Steinschneider gemacht hatte, des Inhalts, daß er mir seine Kopie eines Responsums des Maimondes über die Lebensdauer gezeigt, aber mit Bedauern hinzugefügt habe, daß seine Abschrift fehlerhaft sei, und daß eine bessere Kopie oder das Original nicht zur Verfügung ständen. Auch in seiner «Arabischen Literatur der Juden», die gleichfalls im Jahre 1902 erschienen ist, erwähnt er zwar auf Seite 212 das «Ms. Shapira» und seine Abschrift, sagt aber nicht, wo sich das Manuskript befindet. Sogleich nach Auffinden meiner Notiz versuchte ich, etwas über den Verbleib der Steinschneiderschen Kopie des Responsums zu erfahren. Meine Bemühungen blieben jedoch erfolglos, und ich konnte, nachdem bereits 17 Jahre seit dem Tode von Steinschneider vergangen waren, nicht mehr feststellen, wohin die vergilbten Blätter mit seiner Abschrift, die ich im Jahre 1902 gesehen hatte, gekommen waren. Weder konnte Professor Alexander Marx diese Abschrift in der Bibliothek des Jewish Theologícal Seminary in New York finden, die den wissenschaftlichen Nachlaß von Steinschneider erworben hatte, noch konnte ich sie in der Bibliothek der Jüdischen Gemeinde Berlin entdecken, die eine verhältnismäßig kleine Zahl von Convoluten seiner Notizen besaß. Dieses Mißlingen war jedoch insofern von Nutzen, als es mich dazu bewog, über die von Steinschneider gemachte Abschrift hinaus dem ganzen Codex «Shapira 102» nachzugehen, aus dem die Kopie des Responsums geflossen war. Das Suchen nach dieser seit dem Jahre 1879 nicht mehr erwähnten Handschrift bot aber Schwierigkeiten, da der Händler Shapira alle größeren Bibliotheken und Sammler Europas beliefert hatte und die Nummern 102 und 103 nur dem Zwecke des Verkaufs dienende Buchhändlerbezeichnungen, aber keine festen Standnummern waren, die als sichere Anhaltspunkte hätten dienen können.

Nach einigen Recherchen gelangte ich zu der festen I berzeugung, 
daß das von mir gesuchte Original in dem Ms. Opp. Add. $8^{\circ} 46$ enthalten sei, daa von Ad. Neubauer in seinem «Catalogue of the Hebrew Manuscripts in the Bodleian Library» (Oxford 1886) als Nr. 2497 auf Seite 887 beschrieben ist. Auf Grund der Tatsache, daß nach der Beschreibung Neubauers die Makamen des Tobijja in der Oxforder Handschrift nicht gemäß Steinschneiders Notiz am Anfang von Shapira 102, sondern am Ende des ersten Teiles des jetzigen Oxforder Codex stehen, mußte ich vermuten, daß die beiden Handschriftenbändchen nach dem Ankauf in Oxford in e í n e n Band zusammengebunden woren waren, und zwar in umgekehrter Folge, so daß Shapira 103 den Anfang des jetzigen Codex bildete. Trotzdem hatte ich Bedenken, die mir gewisse Annahme zu äußern, weil es mir unwahrscheinlich erschien, daß weder Steinschneider noch Kaufmann, die über dieses Responsum in den Jahren 1888, 1895 und 1902 geschrieben hatten, nicht nach dem Verbleib des Originals geforscht, sondern sich mit einer in Eile hergestellten, ungenauen Abschrift begnügt haben sollten, obwohl der Neubauersche Katalog, der bereits im Jahre 1886 erschienen war, ihnen zur Verfügung stand. Eine Anfrage an den Direktor der Bodleian Library, den verstorbenen Prof. A. Cowley, brachte jedoch bald Gewißheit. In seiner Antwort vom 3. 9. 1925 bestätigte er mir die Richtigkeit meiner Annahme wie folgt: «I think your very ingenious deduction is right. Unfortunately I cannot find that we have any list of MSS offered for sale by Schapira. Neubauer took no interest in the history and provenance of MSS, and has left no record of the sources from which he obtained them. The volume MS. Opp. add. $8^{\circ} 46$ is evidently composed of two parts bound together. Part 1 bears a label with No. 92 and part 2 has No. 91. These are certainly the dealer's marks. I have had these carefully removed, or rather moved to a blank space, and find under No. 91 in the same (dealer's) hand No. 102, and under No. 92 in pencil 103 written probably in the Library ... The responsum you quote begins on f. $128^{\prime \prime}$ and ends on f. 1311 ... MS. Schapira 103 is evidently, as you conjectured, the first part of MS. Opp. add. $8^{\circ} 46$ (Neubauer's Catal. no. 2497), occupying ff. 1-91. The extracts from the Makamat Tobiya are at the beginning of MS. Schap. 102-that is to say on f. 92 of MS. Opp. add. $8^{\circ} 46$ as now bound. The quotation from MS. Sch. 103 is found on f. $841 \ldots$ » Ich gab daraufhin von dem Resultat meiner Nachforschung sogleich Kenntnis in einem kurzen Artikel «Ein unediertes Responsum 
des Maimondes», der in der «Deutschen Literaturzeitung» vom 25. 9. 1926 erschienen ist, und ich hoffte, daß ich die wichtige Urkunde auf Grund der aus Oxford erbetenen Photographien sehr bald würde herausgeben können. Mehrmaliger Ortswechsel, Wirren und Kriege, Belastung durch ein zeitraubendes Verwaltungsamt und Inanspruchnahme durch die Lehrtätigkeit verzögerten jedoch immer aufs neue meine Arbeit; und nachdem diese Hindernisse zum groBen Teil in Wegfall gekommen waren und ich an die Bearbeitung des Textes denken konnte, begann der auch jetzt noch anhaltende, unerträgliche Zustand des «Waffenstillstandes», der die Jüdische Nationalund Universitäts-Bibliothek von ihren Schätzen auf dem ScopusBerge abschneidet, so da6 ich, ebenso wie alle anderen Jerusalemer, auf meine eigene bescheidene Büchersammlung angewiesen bin sowie auf die Bücher, die Bekannte mir leihen. Ich dürfte daher eigentlich auch jetzt noch nicht Text und Úbersetzung des Responsums herausgeben; denn in dem sogenannten «gelehrten Apparat» werden manche Belegstellen, die für mich jetzt unerreichbar sind, fehlen. Ich glaube aber, eine weitere Verzögerung in der Herausgabe nicht mehr verantworten zu können, und hoffe, daß ich in der Lesung und Übersetzung des Responsums im wesentlichen das Richtige getroffen und in der Erklärung auf die wichtigsten Punkte hingewiesen habe.

Jerusalem, im Oktober 1950.

Bei Beendigung des Druckes ist es mir eine angenehme Pflicht, sowohl dem Verleger, Herrn Dr. H. Karger, meinen Dank auszusprechen, der es sich hat angelegen sein lassen, das Büchlein in würdiger Form erscheinen zu lassen, als auch Herrn Dr. M. PleBner, der mir bei Lesung der Korrekturen mit großer Sachkenntnis geholfen hat.

Jerusalem, im Mai 1952. G. W. 